\title{
TRANSPLANTATION AND PERFUSION OF MICROVASCULAR FRAGMENTS IN A RODENT MODEL OF VOLUMETRIC MUSCLE LOSS INJURY
}

\author{
M. Pilia ${ }^{1}$, J.S. McDaniel ${ }^{1}$, T. Guda ${ }^{1,2}$, X.K. Chen ${ }^{1}$, R.P. Rhoads ${ }^{3,4}$, R.E. Allen ${ }^{4}$, B.T. Corona ${ }^{1}$ and C.R. Rathbone ${ }^{1, *}$
}

\author{
${ }^{1}$ Extremity Trauma and Regenerative Medicine, United States Army Institute of Surgical Research, \\ Fort Sam Houston, TX, USA \\ ${ }^{2}$ Department of Biomedical Engineering, University of Texas at San Antonio, San Antonio, TX, USA \\ ${ }^{3}$ Department of Animal \& Poultry Sciences, Virginia Tech, Blacksburg, VA, USA \\ ${ }^{4}$ Department of Animal Sciences, University of Arizona, Tucson, AZ, USA
}

\begin{abstract}
Few clinical options are available for the treatment of volumetric muscle loss (VML). An important consideration that needs to be addressed for the development of treatments for these injuries is the establishment of a vascular supply sufficient to support skeletal muscle regeneration. The objective of the current study was to evaluate the potential for microvascular fragments (MVFs) harvested from adipose tissue to support tissue perfusion for VML. Tibialis anterior muscle defects in rats were replaced with constructs that were created on the day of surgery containing either (1) collagen only (COL), (2) freshly isolated microvascular fragments in collagen (MVF), or (3) adipose tissue derived stem cells (ASCs) in collagen. Muscles were harvested 7 and 14 days after surgery. Defects treated with MVFs had a vessel density higher than the other groups at both 7 and 14 days, and those treated with ASCs had a higher vessel density than COL by day $14(p<0.05)$. Perfused vessels were observed in both the ASC and MVF treated defects at day 14, as well as at day 7 in the MVF. This study supports the use of MVFs as a platform to improve tissue perfusion to treat large VML defects. The use of freshly isolated MVFs on the day of surgery supports their clinical use and application.
\end{abstract}

Keywords: Microvessel, volumetric muscle loss, skeletal muscle, stem cell, angiogenesis.

*Address for correspondence:

Christopher R. Rathbone

3698 Chambers Pass BLDG 3611

JBSA Fort Sam Houston, TX, 78234, USA

Telephone Number: (210) 539-3670

FAX Number: (210) 539-3877

Email: christopher.r.rathbone.civ@mail.mil

\section{Introduction}

Volumetric muscle loss (VML) occurs with surgical interventions and traumatic injuries; a regenerative standard of care does not exist for these wounds (Grogan and Hsu, 2011). In addition to overt losses of muscle following trauma, the use of muscle flap techniques as a means of soft tissue coverage and to improve bone healing can result in donor site VML (Cierny et al., 1983; Kramers-de Quervain et al., 2001). The development of a successful treatment for VML has significant implications for a large number of patients. For example, wounded service members are unable to return to duty due to the loss in function and weakness resulting from muscle injuries that include VML (Cross et al., 2011).

An insufficient tissue vascular supply may partially explain the impaired myogenesis and consequent fibrosis within large VML defects, an etiology reminiscent of large muscle grafts with slow and/or impaired revascularization (Markley et al., 1978). To improve tissue perfusion and regenerative outcomes in traumatized soft tissues, a variety of biological therapies utilizing growth factors, stem and progenitor cells, and prevascularized constructs created in vitro have been explored (Levenberg et al., 2005; Koffler et al., 2011; Lesman et al., 2011). The primary advantage of a prevascularized scaffold with mature microvascular networks is that there is less of a reliance on vessel ingrowth into an implant, since such scaffolds require only simple inosculation with the host tissue to improve vascular density and accelerate tissue perfusion (Laschke and Menger, 2012). It is important to make the distinction between the use of prevascularized scaffolds containing mature networks and those containing microvascular elements (i.e., initial nascent microvessels or endothelial cell tubes). Although the latter circumvents the need for de novo vessel formation there may be a period of maturation required before tissue perfusion is improved. However, the development of mature prevascularized scaffolds in vitro typically requires significant manipulation including the use of multiple cell types derived from a variety of sources (e.g., endothelial cells, mural cells, stem cells), an increased in vitro culture period, and an additional surgery in the case of in situ prevascularization, making this approach difficult to translate to the clinic (Laschke and Menger, 2012). With regards to skeletal muscle, prevascularization has been demonstrated to be a promising strategy but has been limited to relatively small skeletal muscle defects (Levenberg et al., 2005; Koffler et al., 2011). 

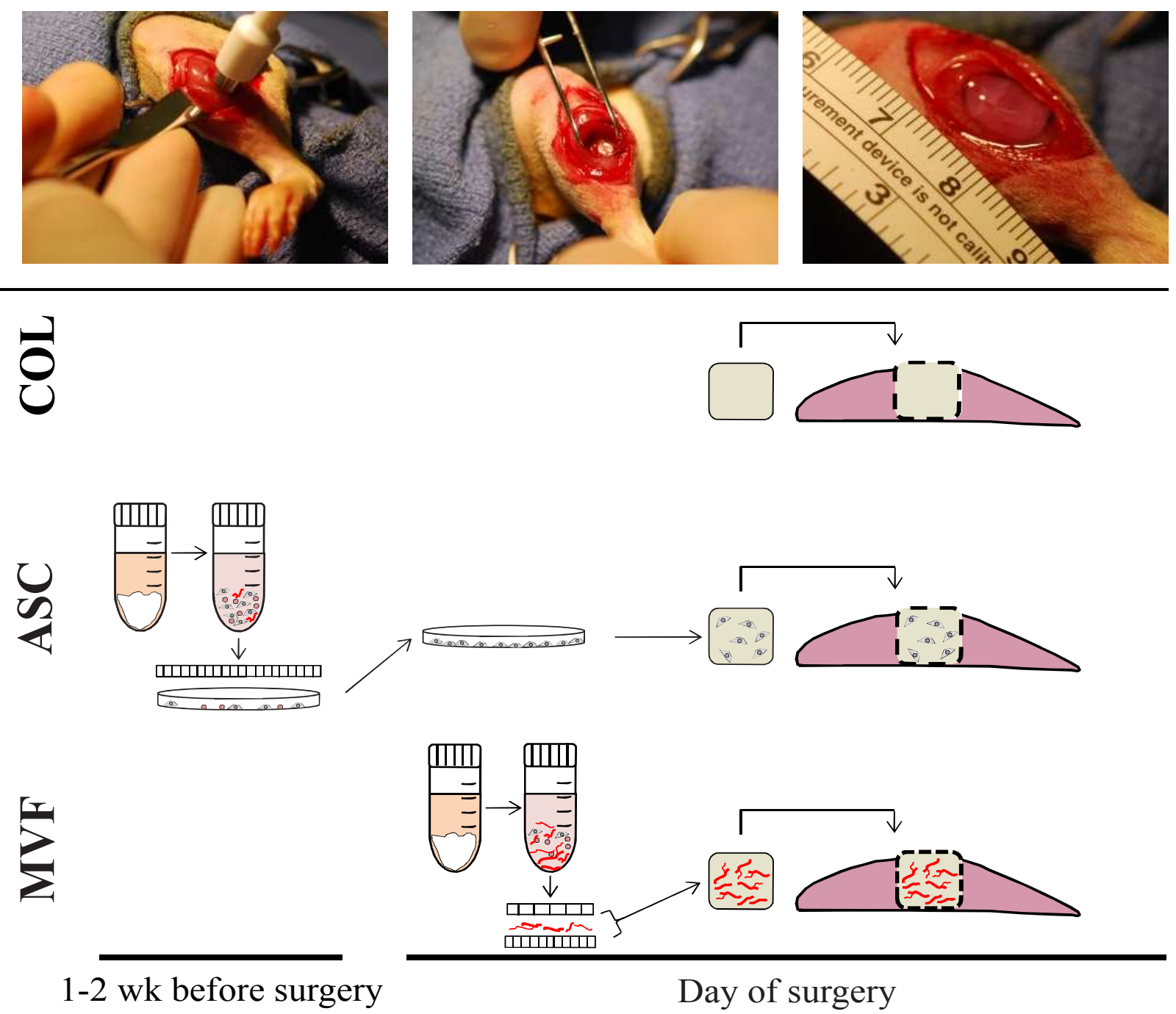

Fig. 1. Top: Images demonstrating the creation of volumetric muscle loss (VML) muscle defect and repair. After blunt dissection and the insertion of a metal plate between the tibialis anterior (TA) and extensor digitorum longus (EDL) muscles, a $6 \mathrm{~mm}$ biopsy punch was inserted deep to the metal plate, and the tissue was excised to create a full thickness muscle defect. Bilateral defects were replaced with one of the constructs containing either collagen only, adipose-derived stem cells (ASC) in collagen, or microvascular fragments (MVF) in collagen. Bottom: Schematic describing experimental design and procedures. 1-2 weeks prior to the surgery (ASC) were isolated using standard procedures, seeded on tissue culture plates, and used as passage 2 cells for implantation. On the day volumetric muscle loss defects were created, MVFs were isolated from adipose tissue, and collagen only (COL), ASCs in collagen (ASC), or MVFs in collagen (MVF) constructs were prepared prior to their implantation into VML defects.

Since microvascular fragments (MVFs) are procured as a heterogeneous mixture of arterioles, capillaries, and venules that are harvested from adipose tissue (Hoying et al., 1996), they bypass the need for de novo microvessel formation. Although time is still required for them to develop mature networks, their ability to circumvent the need for the formation of nascent microvessels may impart an important advantage over a number of strategies. As opposed to the combination of vascular and supporting cells in vitro, MVFs have previously been shown to spontaneously form a mature vascular network even without additional cell or growth factors supplementation (Hoying et al., 1996). Moreover, since stem cells are resident within the microvasculature (Crisan et al., 2008; Ergun et al., 2011), and are associated with MVFs (Laschke et al., 2012), a logical speculation is that cells and growth factors associated with MVFs may help to promote a regenerative environment. Previous in vivo studies have demonstrated the ability of MVFs to improve vascular density and perfusion in vivo (Shepherd et al., 2004; Shepherd et al., 2007; Laschke et al., 2012), and in vitro studies have demonstrated that skeletal muscle satellite cells improve the angiogenic capacity of MVFs (Rhoads et al., 2009). Collectively, this suggests that MVFs may be particularly relevant for skeletal muscle vascularization. Given the need for clinically relevant therapies for VML, it is of utmost importance to identify practical approaches (i.e., autologous therapies with minimal manipulation) that can be applied to challenging injuries (i.e., large muscle defects). Therefore, the purpose of this study was to determine if freshly isolated MVFs are capable of improving the vascular density within VML defects. 

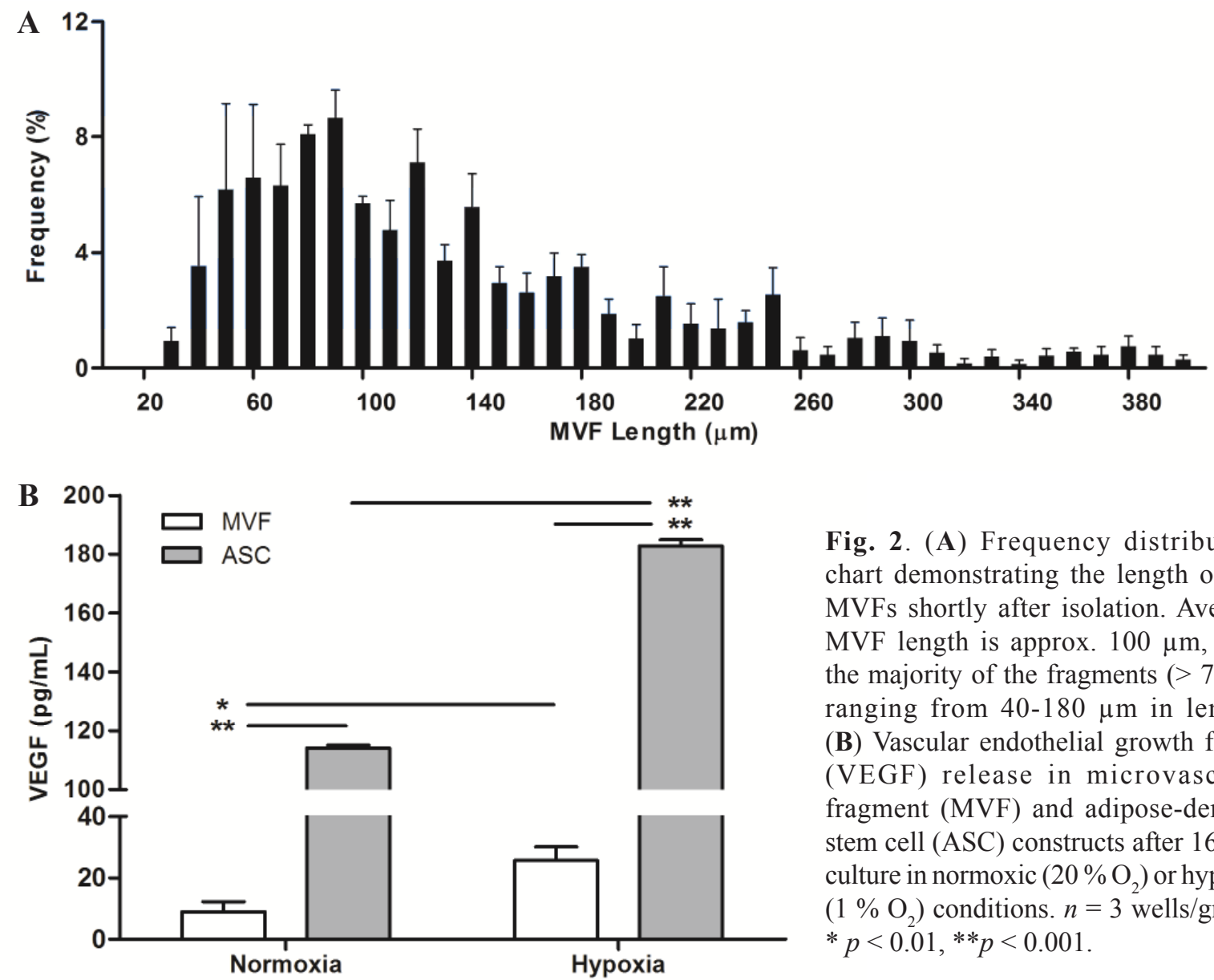

Fig. 2. (A) Frequency distribution chart demonstrating the length of the MVFs shortly after isolation. Average MVF length is approx. $100 \mu \mathrm{m}$, with the majority of the fragments $(>78 \%)$ ranging from $40-180 \mu \mathrm{m}$ in length. (B) Vascular endothelial growth factor (VEGF) release in microvascular fragment (MVF) and adipose-derived stem cell (ASC) constructs after $16 \mathrm{~h}$ of culture in normoxic $\left(20 \% \mathrm{O}_{2}\right)$ or hypoxic $\left(1 \% \mathrm{O}_{2}\right)$ conditions. $n=3$ wells/group. $* p<0.01, * * p<0.001$.

\section{Materials and Methods}

This study has been conducted in compliance with the Animal Welfare Act and the Implementing Animal Welfare Regulations and in accordance with the principles of the Guide for the Care and Use of Laboratory Animals and was conducted in the animal facility at the US Army Institute of Surgical Research. Rats were housed individually in a temperature-controlled environment with a 12-hour light/ dark cycle.

\section{VML injury}

Male Lewis rats (2 to 3 months of age; approx. $350 \mathrm{~g}$; Harlan Laboratories, Indianapolis, IN, USA) were used for VML studies. Bilateral VML defects (Fig. 1) were created by removing the middle portion of both tibialis anterior (TA) muscles of recipient rats using a $6 \mathrm{~mm}$ muscle biopsy punch (Fig. 1) (Acuderm Inc, Ft. Lauderdale, FL, USA). Skin and fascia were separated from the underlying muscle using blunt dissection and a metal plate was inserted underneath the TA and above the extensor digitorum longus (EDL). The biopsy punch was inserted deep to the metal plate. The tissue was excised to create a full thickness muscle defect. Constructs (one of the three described below) were removed from the 48 -well plate and placed into the defect site. The constructs tightly filled the defect area, and the site of injury was marked by placing four knots on the 4 opposite corners by using prolene suture (6-0). The wound was closed by first suturing the fascia back together with simple interrupted vicryl suture, and the skin was stapled closed.

\section{Construct preparation}

A schematic of the methodologies and experimental design is presented in Fig. 1. Tissue defects were replaced with (1) collagen only (COL), (2) adipose-derived stem cells in collagen (ASC), or (3) MVFs in collagen (MVF), and animals were euthanized 7 and 14 days later $(n=5-6 /$ group). All constructs were created on the day of surgery. Tissue defects replaced with MVFs isolated from Green Fluorescent Protein (GFP) animals were treated similarly, and animals were euthanized 7 days after injury $(n=3$ / group). Weight matched controls (CTRL) animals were left untreated. Microvascular fragments were isolated from the epididymal fat pads of wild-type male Lewis rats (approx. 400 g) (Harlan Laboratories, Indianapolis, IN, USA) or GFP transgenic Lewis rats (Rat Resource and Research Center, Columbia, MO, USA) using a technique similar to that developed by Hoying et al. (Hoying et al., 1996). Briefly, adipose tissue from the epididymal fat pads of rats were subjected to a limited (approx. $8 \mathrm{~min}$ ) collagenase digestion $(2 \mathrm{mg} / \mathrm{mL}$ in phosphate buffered saline (PBS) containing $0.1 \%$ bovine serum albumin) (Worthington Biochemical Corporation, Lakewood, NJ, USA), washed, and filtered through $500 \mu \mathrm{m}$ and $30 \mu \mathrm{m}$ filters to remove large debris and single cells, respectively. A subset of MVF isolates $(n=3)$ was used to quantify MVF length by analyzing pictures ( $>200 \mathrm{MVF} /$ isolate) seeded on 
uncoated 24-well plates using ImageJ 1.44p (U.S. National Institutes of Health, Bethesda, MD, USA). The majority of MVFs were between 40 and $180 \mu \mathrm{M}$ in length (> $78 \%$ ) (Fig. 2). MVFs were suspended in cold Type I collagen ( $3 \mathrm{mg} / \mathrm{mL}$; BD Biosciences, Bedford, MA, USA) prepared with Dulbecco minimum essential medium (DMEM) (1x final) at a density of 40-50,000 MVFs $/ \mathrm{mL}$ and seeded into individual wells of 48 -well plates $(0.4 \mathrm{~mL})$. Constructs were placed in the incubator $\left(37^{\circ} \mathrm{C}\right)$ for $20 \mathrm{~min}$ to polymerize after which an equal volume of $10 \%$ Fetal Bovine Serum (FBS)/DMEM was added to each well until implantation $(<3 \mathrm{~h})$. Adipose derived stem cells were isolated from the epididymal fat pads of wild-type male Lewis rats (approx. $400 \mathrm{~g})$ according to standard ASC isolation procedures (Zuk et al., 2001). Briefly, finely minced adipose tissue was digested for a period of $45 \mathrm{~min}$ and filtered through $30 \mu \mathrm{m}$ filters after the removal of floating adipocytes. Filtered cells were seeded on tissue culture dishes and passaged at approx. $70 \%$ confluency using trypsin-EDTA solution. At the second passage, ASCs were suspended in cold collagen as described above at a density of $5 \times 10^{5}$ ASCs $/ \mathrm{mL}$ and seeded into individual wells of 48 -well plates $(0.4 \mathrm{~mL})$. The purity of ASCs was confirmed in a subset of cultures by positive expression for MSC markers (CD90, CD105, CD73), negative expression for CD45, and multi-differentiation potential (osteogenic, neurogenic, adipogenic) when cultured in the appropriate induction media (data not shown). Empty collagen constructs were prepared exactly as described for MVFs and ASCs with the exception that neither were added.

\section{Vascular Endothelial Growth Factor release}

MVFs and ASC constructs were created as described above and placed in a humidified incubator at ambient $\mathrm{O}_{2}$ (normoxia; $20 \%$ ). Twenty-four hours later medium was changed to serum free medium (DMEM containing $100 \mathrm{U} /$ $\mathrm{mL}$ each of penicillin and streptomycin (Life Technologies, Grand Island, NY, USA). Plates in the normoxic condition were returned to the ambient $\mathrm{O}_{2}$ incubator and plates to be treated with hypoxia were transferred to a humidified incubator set to $1 \% \mathrm{O}_{2}$. Sixteen hours later medium was removed ( $n=3$ /group) and analyzed for Vascular Endothelial Growth Factor release by enzyme linked immunosorbent assay (ELISA) by an ELISA kit (R\&D Systems, Minneapolis, MN, USA) to measure VEGF release into the culture medium.

\section{DiI perfusion}

Seven and 14 days after the injury, animals were perfused using 1,1'-Dioctadecyl-3,3,3',3'-tetramethylindocarbocyanine perchlorate (DiI) fluorescent dye (Sigma-Aldrich, St. Louis, MO, USA) following a previously described protocol (Li et al., 2008) to visualize possible inosculation of the implants with the host tissue. Following euthanasia through intraperitoneal pentobarbital sodium injection, the animal's heart was exposed, and PBS was used to flush the circulation via cardiac puncture. This was followed by subsequent perfusions with $\mathrm{DiI}$ in $\mathrm{PBS} / 5 \%$ glucose and $4 \%$ paraformaldehyde in $0.2 \mathrm{M}$ phosphate buffer solution.

\section{Tissue processing and histological procedures}

Tissues were excised, weighed, embedded in a talcumbased gel, and flash frozen in 2-methylbutane chilled in liquid nitrogen $\left(-100^{\circ} \mathrm{C}\right)$. Longitudinal sections, 10 and $30 \mu \mathrm{m}$ thick for standard and confocal microscopy, respectively, were cut using a cryostat $\left(-20^{\circ} \mathrm{C}\right)$. The entire muscle was sectioned and all sections were stored at $-80{ }^{\circ} \mathrm{C}$ until staining. The regions of muscle containing the largest defect area were identified by composite images (40x original magnification) of sections throughout the muscle stained with hematoxylin and eosin (H\&E) or Wheat Germ Agglutinin (WGA), Alexa Fluor ${ }^{\circledR} 488$ conjugate (1:50 dilution, Invitrogen, Carlsbad, CA, USA) to stain for cellular membranes. Vessels were visualized by staining sections with Griffonia simplicifolia (GS)-1 Lectin (Vector Labs) for the identification of endothelial cells and microvessels in vivo (Shepherd et al., 2004; Nunes et al., 2010). To quantify vessel density, composite images were created as described above and the entire defect area or non-overlapping fields of view of the muscle outside the defect area were analyzed using ImageJ 1.44p (U.S. National Institutes of Health). Images were converted to grayscale, binarized, skeletonized, and superimposed on a grid containing $40 \mu \mathrm{m}$ long squares to count vessel density, as previously described (Rieder et al., 1995). At least two sections per muscle separated by at least $500 \mu \mathrm{m}$ were analyzed. Perfused vessels were identified by superimposing DiI and GS-1 Lectin stained images using both conventional (10 $\mu \mathrm{m}$ sections) and confocal fluorescent microscopy (30 $\mu \mathrm{m}$ sections). Sections were also stained for sarcomeric myosin (MF20: 1:10 dilution, Developmental Studies Hybridoma Bank, Iowa City, IA, USA) or endothelial cells (CD31: 1:100, BD Biosciences, Bedford, MA, USA) with corresponding Alexa Fluor ${ }^{\circledR}$ 488 labeled secondary antibodies (1:500), Invitrogen). Tissue containing MVFs isolated from GFP transgenic animals were sectioned as described above, fixed with $4 \%$ paraformaldehyde, and stained using anti-GFP antibody (1:100 dilution) with an Alexa Fluor ${ }^{\circledR} 488$ secondary (1:500 dilution, Invitrogen). All sections containing fluorescent stains were mounted with media containing 4',6-diamidino-2-phenylindole (DAPI) (Invitrogen) to visualize nuclei.

\section{Statistical analyses}

SigmaPlot $^{\circ} 12.0$ (Systat Software, San Jose, CA, USA) was used to run Analysis of Variance tests with Student Newman-Keuls comparison analysis to determine differences between the experimental groups and time points. Statistical significance was determined when $p<0.05$. All results are presented as mean \pm standard error of the mean (SEM).

\section{Results}

\section{Vascular Endothelial Growth Factor release}

The amount of VEGF released increased 2.8- and 1.6-fold when cultured in response to hypoxia for MVFs and ASCs, 
$\mathrm{H} \& \mathrm{E}$

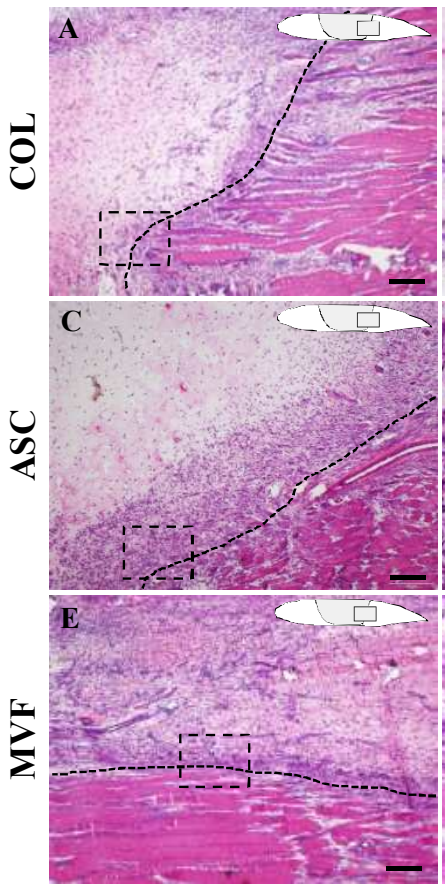

$\mathrm{H} \& \mathrm{E}$
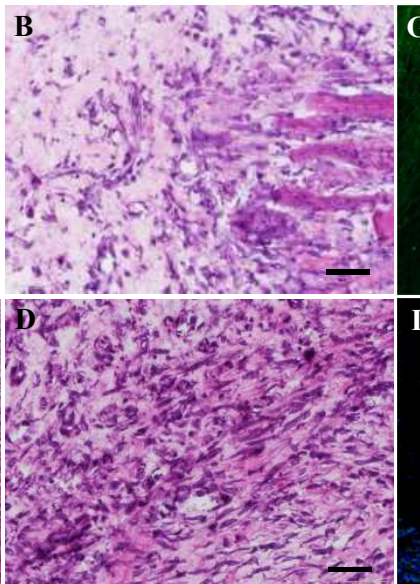
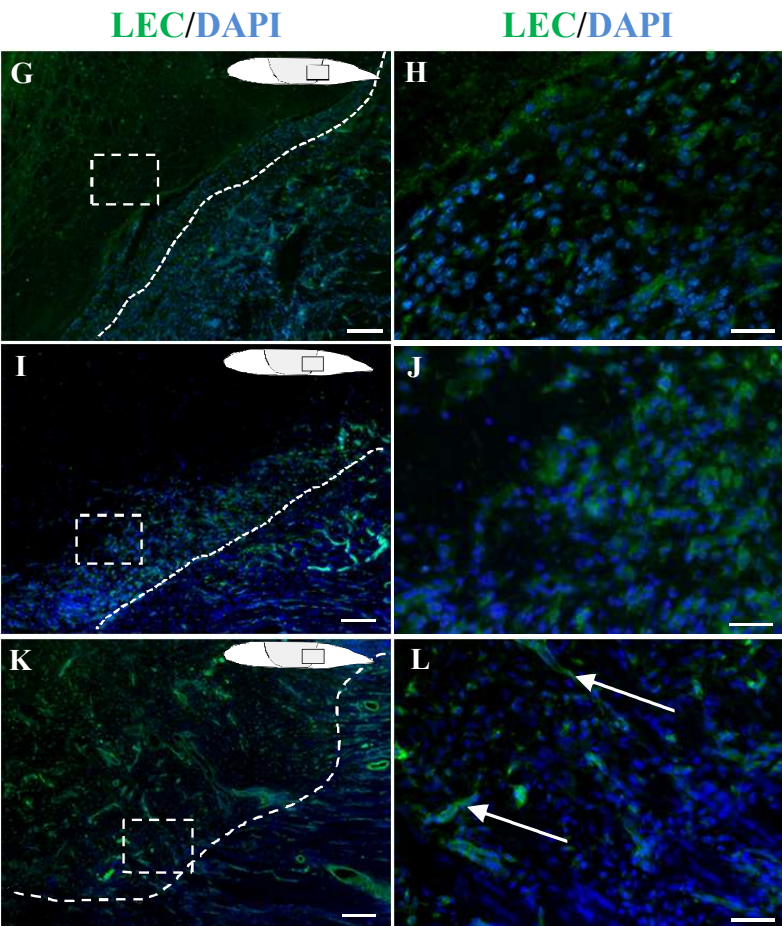

Fig. 3. Longitudinal sections containing the defect-muscle interface stained with hematoxylin and eosin (H\&E) (A-F) or GS-Lectin (green) and DAPI (blue, G-L) to label vessels and nuclei, respectively, 7 days after injury. H\&E and Lectin/DAPI stained images were from adjacent sections from the same muscles. The schematic of the TA longitudinal section (inset) illustrates where the images for each group were captured. Figures $\mathbf{B}, \mathbf{D}$ and $\mathbf{F}$ are magnified images of the boxed areas from $\mathbf{A}, \mathbf{C}$ and $\mathbf{E}$, respectively. Figures $\mathbf{H}, \mathbf{J}$ and $\mathbf{L}$ are magnified images of the boxed areas from $\mathbf{G}$, $\mathbf{I}$ and $\mathbf{K}$, respectively. Hatched line denotes the defect interface. There were several vascular elements (LEC positive) in all groups within the interface 7 days after surgery. The MVF was the only group where blood vessels were seen inside the defect (white arrows, L). A, D, G, G, I and K scale bar $=200 \mu \mathrm{m}$; $\mathbf{B}, \mathbf{D}, \mathbf{E}, \mathbf{H}, \mathbf{J}$ and $\mathbf{L}$ scale bar $=50 \mu \mathrm{m}$.

Table 1. Defect and muscle weights. * indicates significantly less than control $(p<0.001)$ within 7 or 14 days. TA $=$ Tibialis anterior, $\mathrm{EDL}=$ extensor digitorum longus, $\mathrm{CTRL}=$ control, $\mathrm{COL}=$ collagen only, $\mathrm{ASC}=\mathrm{ASC}$ in collagen, $\mathrm{MVF}=$ microvascular fragments in collagen.

\begin{tabular}{|c|c|c|c|c|}
\hline Day & Group & Defect Weight (mg) & TA weight (mg) & EDL weight (mg) \\
\hline \multirow{3}{*}{7} & CTRL & - & $624.8 \pm 17.7$ & $151.1 \pm 1.7$ \\
\hline \multirow{2}{*}{7} & COL & $120.2 \pm 5.5$ & $504.9 \pm 28.6^{*}$ & $130.5 \pm 10.2$ \\
\cline { 2 - 5 } & ASC & $115.0 \pm 8.4$ & $469.6 \pm 14.8^{*}$ & $148.5 \pm 5.3$ \\
\cline { 2 - 5 } & MVF & $117.9 \pm 4.7$ & $494.2 \pm 10.9^{*}$ & $129.2 \pm 12.6$ \\
\hline \multirow{3}{*}{$\mathbf{1 4}$} & COL & $108.9 \pm 4.6$ & $474.0 \pm 24.3^{*}$ & $153.7 \pm 7.1$ \\
\cline { 2 - 5 } & ASC & $104.7 \pm 7.1$ & $495.2 \pm 19.0^{*}$ & $153.2 \pm 8.2$ \\
\cline { 2 - 5 } & MVF & $107.9 \pm 4.6$ & $466.3 \pm 24.8^{*}$ & $144.4 \pm 2.6$ \\
\hline
\end{tabular}

respectively $(p<0.05)$ (Fig. 2). VEGF levels were higher in ASCs than MVFs during both normoxic and hypoxic conditions $(p<0.001)$ (Fig. 2).

\section{Muscle mass}

The weight of the defect removed during surgery represented $17-19 \%$ of the TA mass of an animal of similar size (weight matched control), and was not different among groups $(p>0.05)$ (Table 1). There were no differences among the TA and EDL wet weights among the treatment groups $(p>0.05)$. TA wet weights were significantly less than control at both 7 and 14 days after surgery $(p<0.001)$ (Table 1). The EDL wet weight of animals in the experimental groups was not different from control $(p>0.05)$.

\section{Histological observations}

H\&E staining demonstrated a significant void where the defect was created (center of muscle), the presence of remaining healthy tissue surrounding the defect, and a clear interface where presumptive regeneration and remodeling is active (edge of the defect; Fig. 3). Within the interface vascular elements were present (vessels and endothelial cells) in all groups as demonstrated with GS-1 Lectin staining 7 days after injury (Fig. 3). A notable presence of vascular elements clearly within the defect area juxtaposed to the interface was present only in the MVF group (Fig. 3).

The main purpose of this study was to evaluate vascularity and angiogenic activity within the defect. Although there was little evidence of cellular infiltration in collagen implants 7 days after injury (Figs. 4A-B, 5C- 


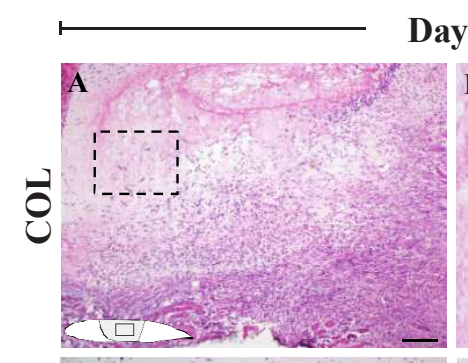

Day 7
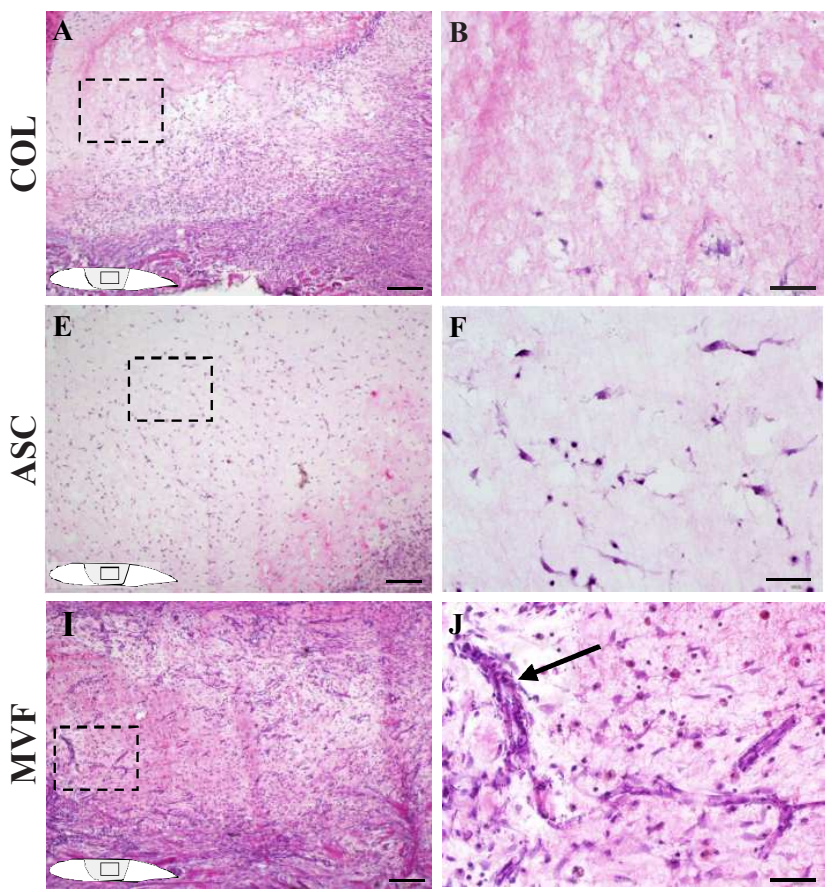

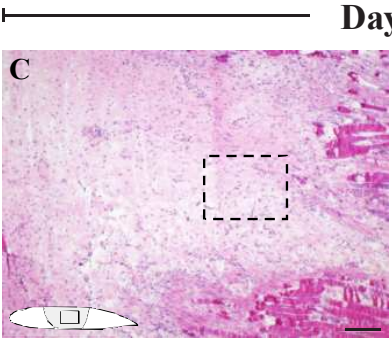

Day 14
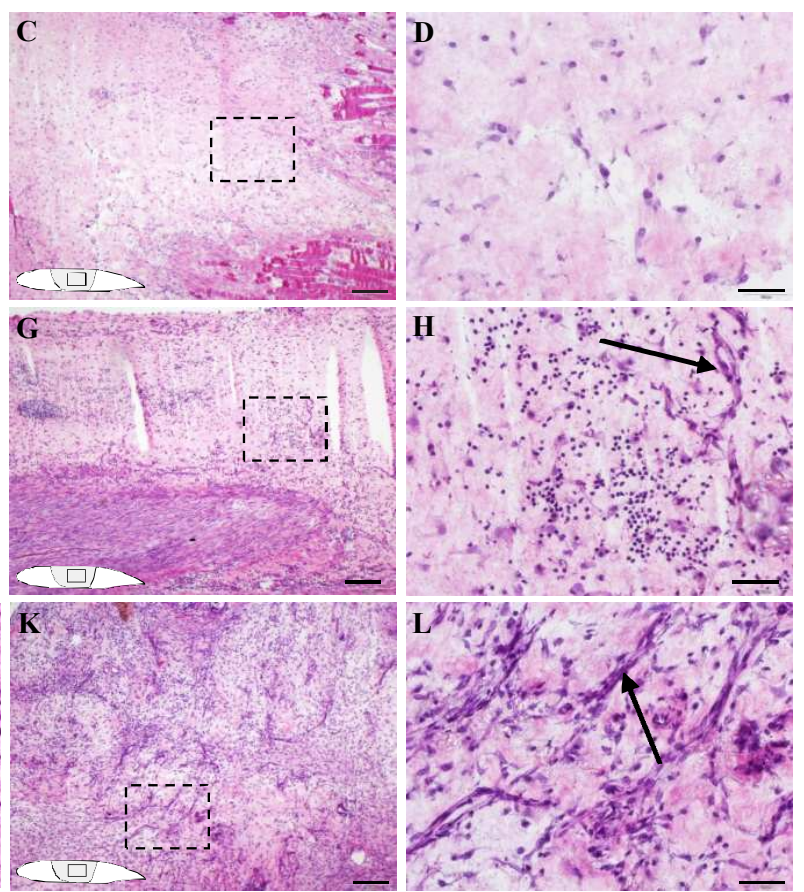

Fig. 4. Longitudinal sections within the muscle defect stained with hematoxylin and eosin (H\&E). The schematic of the TA longitudinal section (inset) illustrates where the images for each group were captured. Figures B, F, J, D, H and $\mathbf{L}$ are magnified images of the boxed areas from $\mathbf{A}, \mathbf{E}, \mathbf{I}, \mathbf{C}, \mathbf{G}$ and $\mathbf{K}$, respectively. Muscles containing defects that received collagen (COL; A-D), adipose-derived stem cell (ASC; E-H) and microvascular fragment (MVF; I-L) implants were evaluated 7 and 14 days after injury. Arrows denote vessels. A, E, I, C, G and $\mathbf{K}$ scale bar $=200 \mu \mathrm{m}$; $\mathbf{B}, \mathbf{F}, \mathbf{J}, \mathbf{D}, \mathbf{H}$ and $\mathbf{L}$ scale bar $=50 \mu \mathrm{m}$.

D), muscles receiving ASC implants had several nuclei (Fig. 4E-F), of which some were positive for GS-Lectin staining (Figs. 5E-F). The overall lack of cells within the COL group but presence of cells within the ASC group that are GS-1 lectin and CD31 positive (Fig. 5F), suggests that some of the implanted ASCs may have undergone endothelial differentiation in vivo, consistent with the endothelial differentiation of ASCs when injected into an ischemic hind limb (Cao et al., 2005). In addition to a high nuclear density, defects receiving MVFs were distinct from the other groups because within the defect area several presumptive vessels were identified with H\&E (Fig. 4IJ) and several GS-1 lectin positive vessels were present at 7 days after surgery (Fig. 5G-H). Unlike the collagen group, the nuclear density and presence of small vessels increased dramatically in the ASC group by 14 days (Figs. 4C-D, G-H and 5I-J, M-N). Interestingly, the high vascular density observed at 7 days was not only sustained but also increased between 7 and 14 days in the MVF group (Fig. $4 \mathrm{~K}-\mathrm{L}$, and 5Q-R). Gross observations of the excised muscle consistently supported the histological measurements. The MVF-implanted muscles had a pink/red color in the defect area whereas there was a white/pale color in the other groups, suggestive of improved perfusion within the defect for MVFs. Immature muscle fibers were indicated by the presence of myosin heavy chain positive fibers in defects in the MVF group (Fig. 5S-T), but were absent in both COL (Fig. 5K-L) and ASC (Fig. 5O-P). Notably, the immature fibers were found to be in close proximity to the vessels.

\section{Vessel density}

The vessel density within COL implants within the defect area was less than MVF but not ASC at 7 days $(p<0.001)$ (Fig. 6A). There was an increase in vascularity between 7 and 14 days for the ASC, and the vessel density in the defect area within the ASC group was greater than COL at 14 days $(p<0.01)$ (Fig. 6A). The vessel density within the defect area for MVF implants was higher than both COL and ASC at both 7 and 14 days after surgery $(p<0.01)$ (Fig. $6 \mathrm{~A})$. Despite the large increases in vessel density with both the ASC and MVF groups, the vessel density in the defect area was significantly less than control muscle at both 7 and 14 days after injury (Fig. 6A). When considering the overall functional capacity of the muscle, the effects on the remaining muscle mass should be considered given that the muscle's functional capacity is governed not only by the changes within the defect but alterations in the rest of the muscle (Corona et al., 2013b). Vessel density was also measured in the area surrounding the muscle defect to determine if the changes in vascularity within the muscle defect had an effect on the surrounding vasculature, but there were no differences among the control muscle and any of the treatment groups (Fig. 6B). However, these results should be interpreted with caution given that changes in muscle fiber size can skew vessel density measurements 

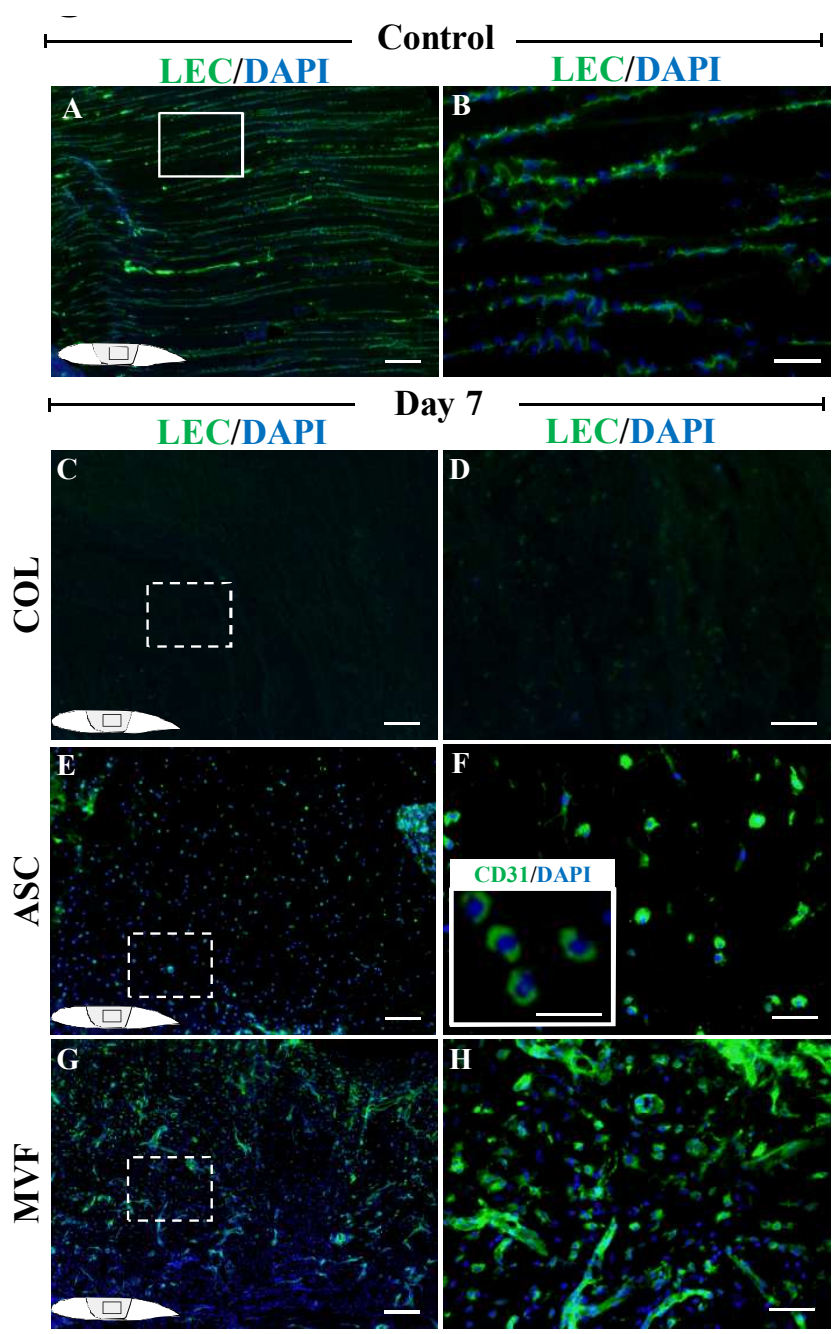

Fig. 5. Longitudinal sections within the muscle defect stained with Lectin to label vessels (LEC, green or red) DAPI to label nuclei (blue), MF20 to label sarcomeric myosin (Myosin, green), or CD31 to label endothelial cells (green). Lectin/DAPI and Myosin/DAPI or Myosin/Lectin stained images were from adjacent sections from the same muscles. The schematic of the TA longitudinal section (inset) illustrates where the images for each group were captured. Control healthy muscle (A-B) and muscles containing defects that received collagen $(\mathrm{COL} ; \mathbf{C}-\mathbf{D}$, I-L), adipose-derived stem cell (ASC; E-F, M-P) and microvascular fragment (MVF; G-H, Q-T) implants were evaluated 7 and 14 days after injury. F inset: adjacent section from the same tissue stained for CD31 (scale bar $=25 \mu \mathrm{m}$ ). Solid arrows denote vessels. Dashed arrows denote immature muscle fibers. A, C, E, G, I, $\mathbf{M}$ and $\mathbf{Q}$ scale bar $=200 \mu \mathrm{m}$; $\mathbf{B}, \mathbf{D}, \mathbf{F}, \mathbf{H}, \mathbf{J}, \mathbf{N}, \mathbf{R}, \mathbf{K}, \mathbf{L}, \mathbf{O}, \mathbf{P}, \mathbf{S}$ and $\mathbf{T}$ scale bar $=50 \mu \mathrm{m}$.

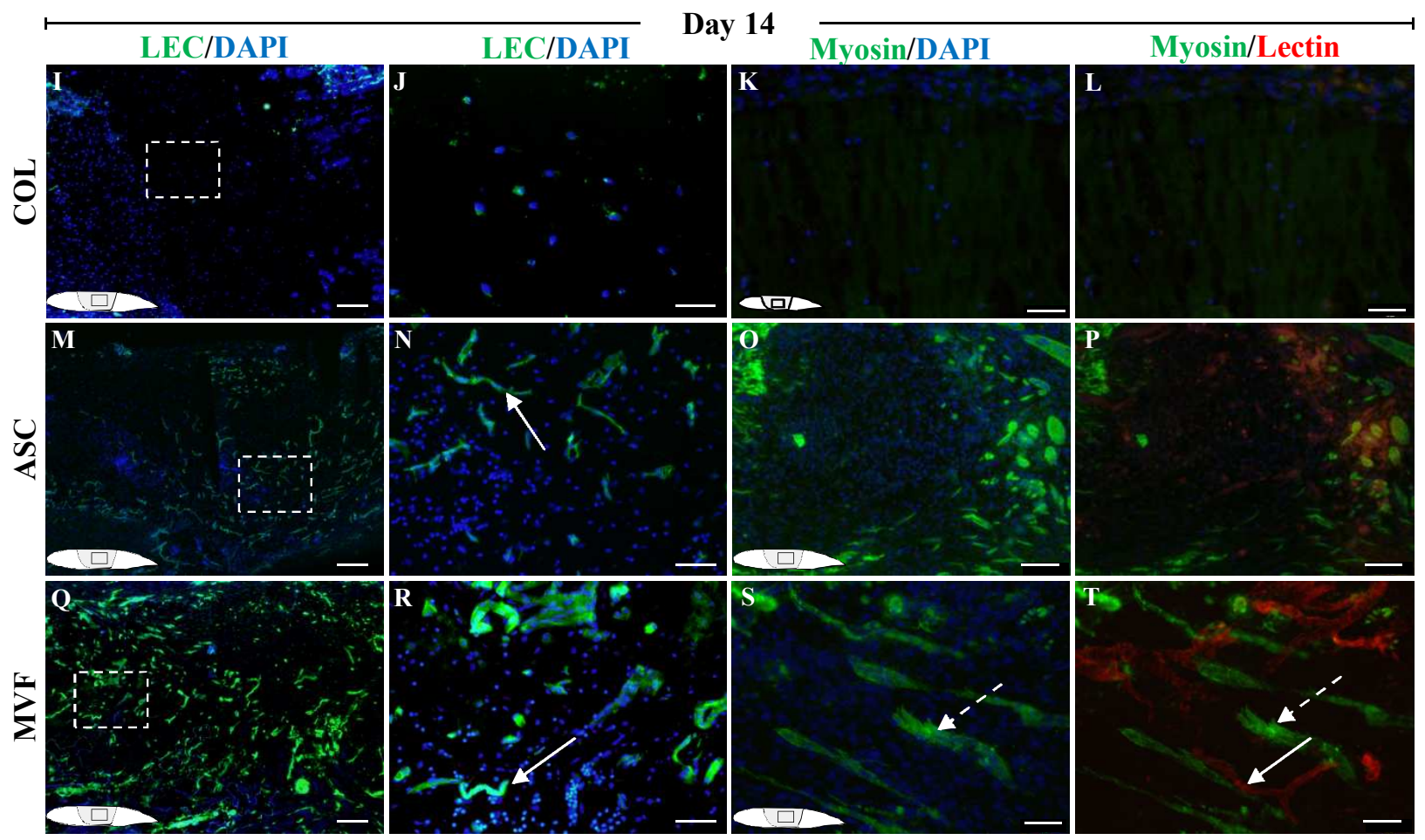



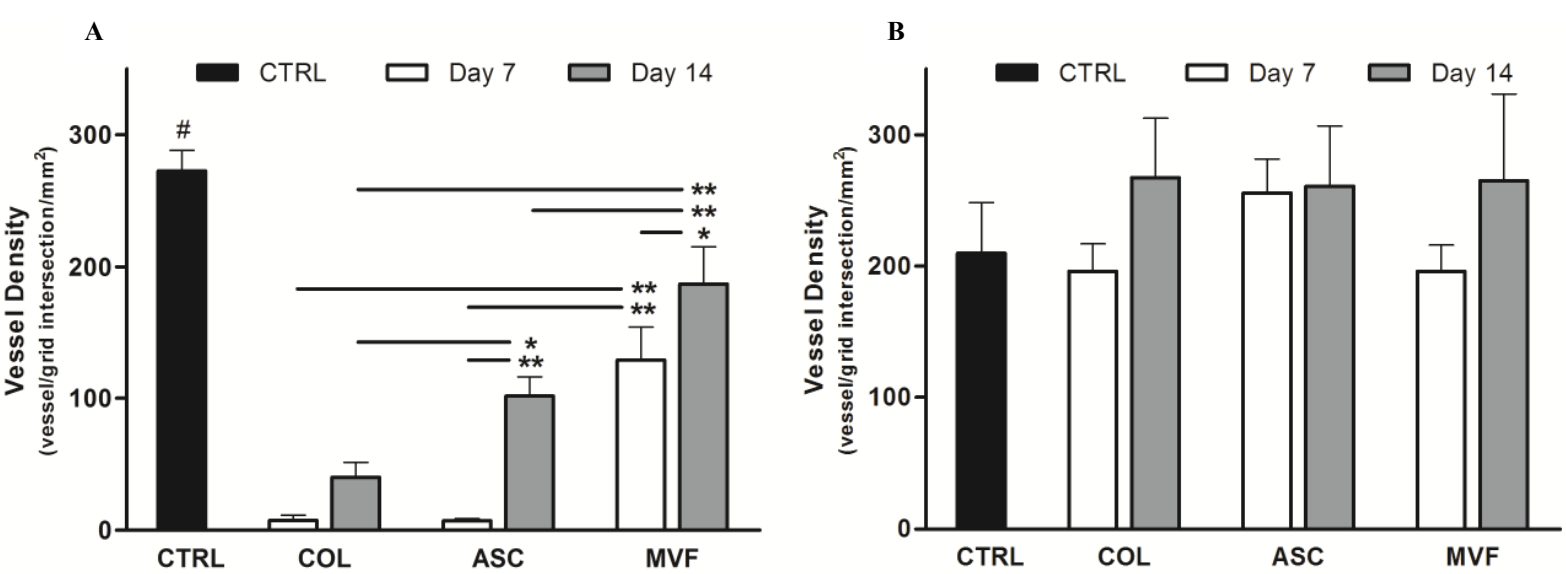

Fig. 6. Quantification of vessel density within the defect $(\mathbf{A})$ or outside the defect in the remaining muscle (B) in control (CTRL) muscles or those receiving collagen (COL), adipose-derived stem cell (ASC), or microvascular fragment (MVF) implants harvested 7 or 14 days after injury. Vessel density analyzed in CTRL $(n=3)$ muscles was in an area consistent with the area of muscle defect for experimental groups ( $n=5-6$ muscles/group/time point). * $p<0.01, * * p<0.001$, \# different from all groups $(p<0.01)$.

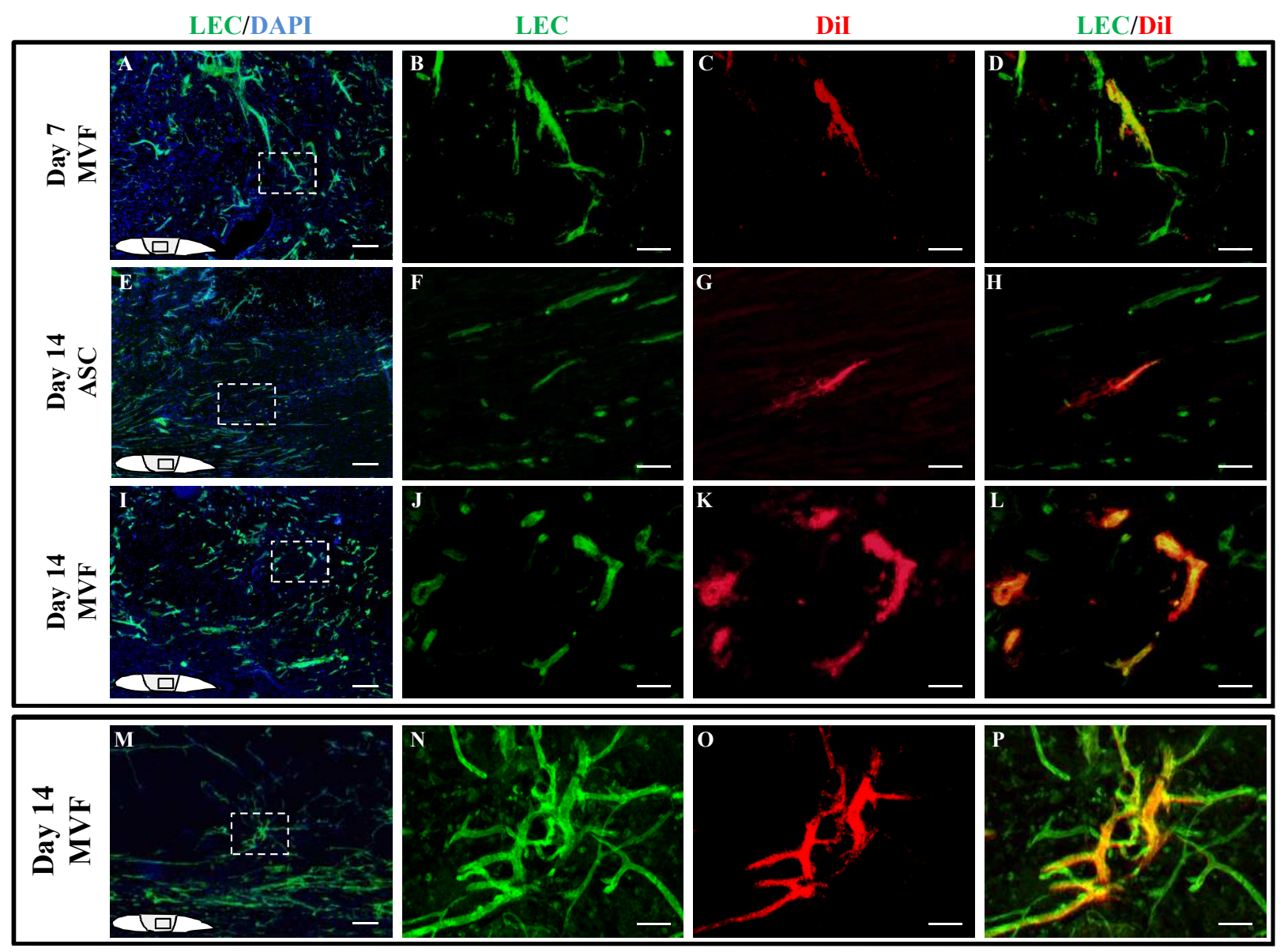

Fig. 7. Longitudinal sections within the muscle defect stained with Lectin (LEC, green) and DAPI (blue) and visualized for 1,1'-Dioctadecyl-3,3,3',3'-tetramethylindocarbocyanine perchlorate (DiI) (red) to label vessels, nuclei, and perfused vessels, respectively. Muscles receiving adipose-derived stem cell (ASC), or microvascular fragment (MVF) implants 7 (A-D) or 14 days (E-P) after injury. The schematic of the TA longitudinal section (inset) illustrates where the images for each group were captured. Figures B-D, F-H, J-L and N-P are magnified images of the boxed areas from A, E, I and M, respectively. A, E, I and $\mathbf{M}$ scale bar $=200 \mu \mathrm{m} . ; \mathbf{B}-\mathbf{D}, \mathbf{F}-\mathbf{H}, \mathbf{J}-\mathbf{L}$ and N-P scale bar $=50 \mu \mathrm{m}$. 


\section{GFP}
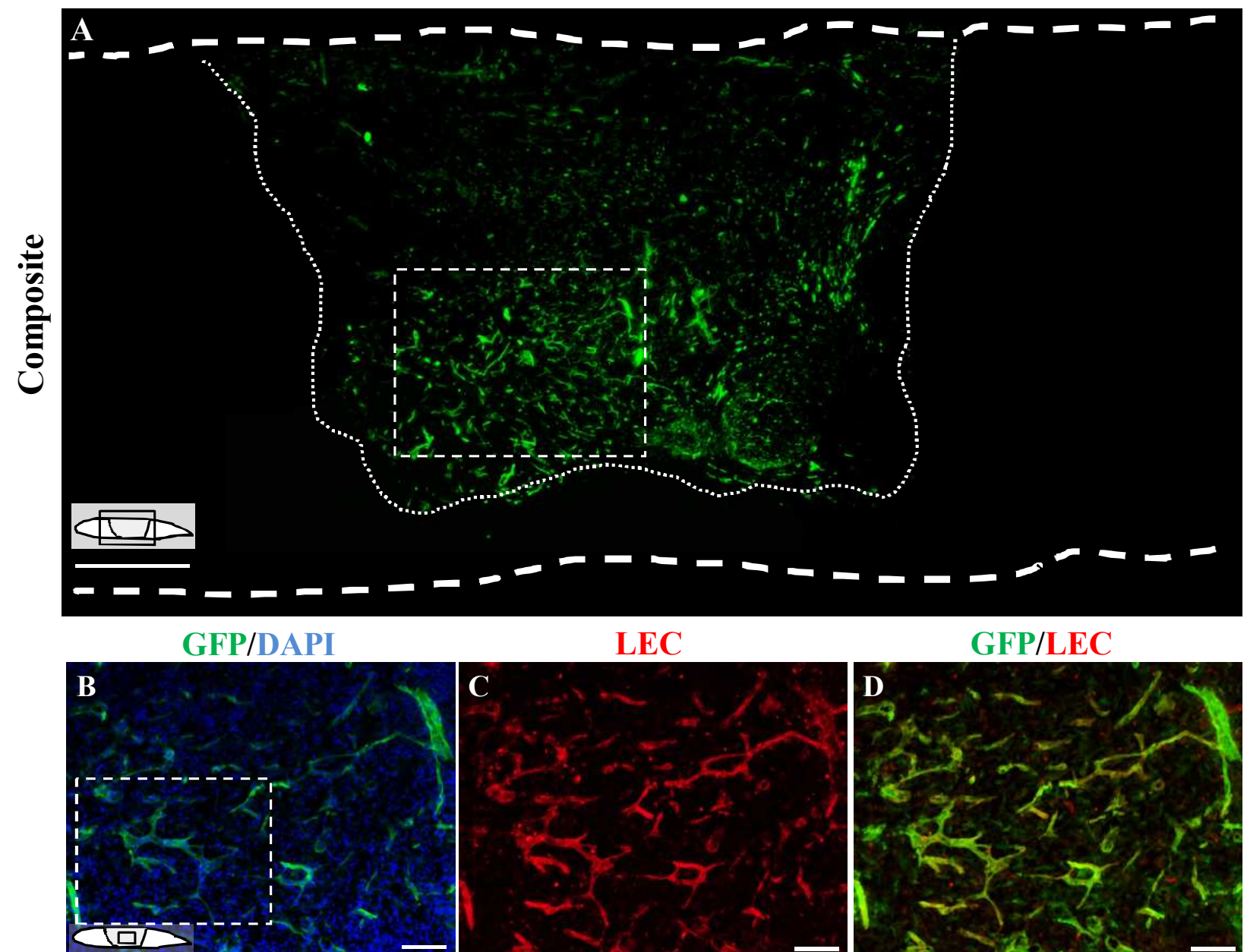

LEC
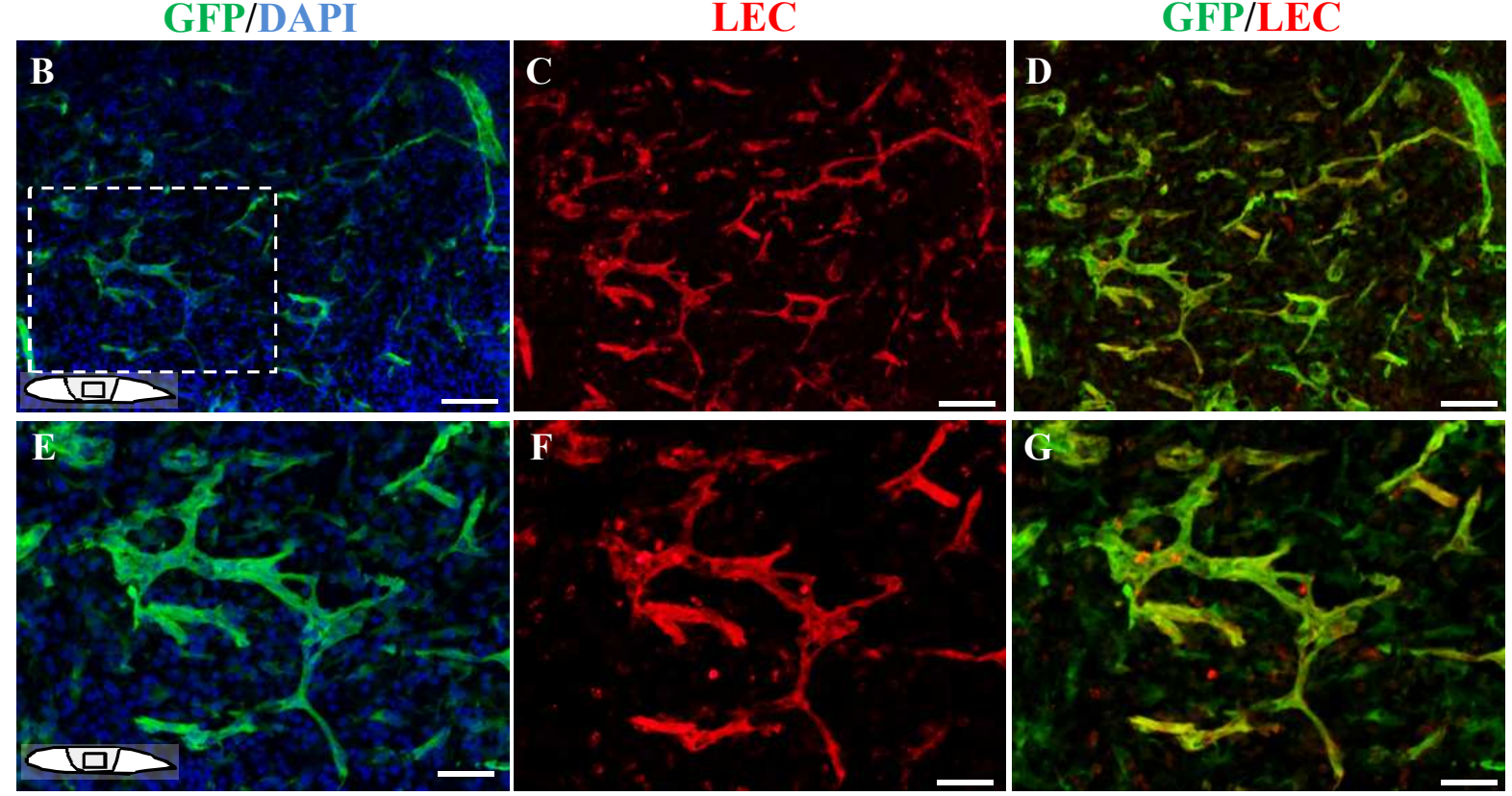

Fig. 8. Longitudinal sections of the muscle defect ( 7 days after injury) that received MVFs isolated from GFP transgenic animals. Sections were visualized for GFP (green), or stained with Lectin (LEC, red) to label the transplanted MVFs and vessels, respectively. (A) Composite photograph (40x original magnification) of the muscle defect. White dotted bar delineates the margins of the muscle. (B-J) Longitudinal sections of the muscle defect visualized for GFP (green), stained with DAPI (blue) or GS-Lectin (LEC, red) to label the transplanted MVFs, nuclei, and vessels, respectively. Figures B-D and E-G are magnified images of the boxed areas from $\mathbf{A}$, and $\mathbf{B}$, and $\mathbf{E}$, respectively. The schematic of the TA longitudinal section (inset) illustrates where the images for each group were captured. Hatched line (A) denotes the border of the muscle section. Dotted line (A) denotes the defect interface. (A) scale bar $=1000 \mu \mathrm{m},(\mathbf{B}-\mathbf{D})$ scale bar $=200 \mu \mathrm{m},(\mathbf{E}-\mathbf{G})$ scale bar $=100 \mu \mathrm{m}$.

(Linderman et al., 2000) and muscle fiber size changes are frequently observed after an injury (Deveci et al., 2001).

\section{Vessel perfusion}

To determine if vessels within the defect area were inosculated with the host, tissue sections were visualized for the presence of DiI, a fluorescent dye that was injected systemically at the time of euthanasia. Fluorescent microscopy demonstrated the inosculation of vessels within the defect area of the MVF group with the host tissue as shown by the presence of DiI within GS-Lectin positive vessels 7 days after surgery (Fig. 7A-D). Perfused vessels identified in the ASC group 14 days after implantation agrees with the increased vessel density observed at 14 days (Figs. 5M-N, 6A, and 7E-H). Vessel perfusion was maintained at 14 days with the MVFs as demonstrated by 
GS-lectin positive vessels and developed networks using both conventional (Fig. 7I-L) and confocal (Fig. 7M-P) microscopy. Although the DiI perfusion demonstrated the capacity for inosculation with the host, the overall level of perfusion was low and was not distributed evenly throughout the defect.

\section{GFP MVF transplantation}

MVFs derived from GFP transgenic animals were transplanted into VML defects to determine if the vascularity we observed was a result of the transplanted vessels or was due to vessel ingrowth. GFP MVFs that were implanted in the VML defect were maintained within the implant and created a very thick interconnected vascular network 7 days after surgery (Fig 8), similar to that observed with MVFs isolated from wild-type animals (Fig. 5). The implanted vessels were clearly identified within the defect area (Fig. 8A). Virtually all of the GFP vessels within the defect were positive for GS-Lectin (Fig. $8 B-G)$. At the interface, there were few vessels/cells in the defect that were $\mathrm{GFP}^{-} / \mathrm{Lectin}^{+}$, and $\mathrm{GFP}^{+} /$Lectin $^{+}$structures were rarely observed outside of the defect (Fig. 8). The observation that GFP negative vessels were rarely found within the defect suggests that the improved vascularity was primarily a result of the transplanted MVFs.

\section{Discussion}

The critical importance of blood flow within healing tissues is well accepted, therefore, improving vascularity is the primary focus for the treatment of a variety of injuries. The current number of treatment strategies being developed and the clinical options available for the treatment of VML is far fewer than for other musculoskeletal injuries (e.g., fracture, non-union). Of these, few strategies are geared towards improving tissue perfusion, especially those that can be used in a same-day autologous approach. At the very least, the improvement of vascularity has the potential to augment other approaches where a myogenic source is supplied (Rossi et al., 2011; Corona et al., 2013a; Li et $a l ., 2014)$. To this end, the primary objective of the current study was to determine if the application of freshly isolated MVFs improves vascularity within a VML defect in the early phase following the injury. In the current study we observed a $\sim 20$ fold higher vessel density within the defect of the MVF group, as compared to the COL or ASC groups at the earliest time point studied (Fig. 6). The observation that vessels in the MVF group were perfused by 7 days (Fig. 7) is in congruence with the idea that the use of MVFs may be a means to improve the delivery of oxygen, stem cells, and growth factors for subsequent tissue repair. The fact that MVFs are derived from adipose tissue, the minimization of in vitro manipulation, and the application of the MVFs on the same day of surgery, all support the clinical relevance of this approach.

ASCs are often used in tissue engineering applications (Mizuno, 2010) due, at least in part, to their ability to form vascular networks (Planat-Benard et al., 2004) and positively influence muscle repair (Park et al., 2012; Hwang et al., 2013), making ASCs an excellent comparison tool for the study of MVFs. Consistent with previous findings where ASCs improved vessel density in a laceration model (Hwang et al., 2013), in the current study ASCs improved vessel density within VML defects by 14 days after implantation. However, MVFs outperformed ASCs both qualitatively and quantitatively in terms of vascularization (Figs. 3-6). There are some similarities between the two groups worth noting. Recent studies have suggested that the stem cells, to include ASCs, are derived from the vasculature (Crisan et al., 2008; Ergun et al., 2011). MVFs are a mixture of microvessels, therefore, it is intuitive that MVFs contain resident stem cells (Laschke et al., 2012). Further, it is worth considering that MVF-resident stem cells may have some characteristics in common with ASCs. ASCs improve vascularity either by forming vessels de novo (Planat-Benard et al., 2004) or by improving angiogenesis from surrounding tissue (Jabbarzadeh et al., 2008). Since ASCs were capable of improving vascularity by 14 days after injury, it is tempting to speculate that the stem cells resident on the MVFs may have provided an additional source of stem cells for continued angiogenesis. This is consistent with the increase in vascularity observed with MVFs between days 7 and 14 (Fig. 6). Despite these factors, it is important to emphasize that the most distinct benefit to using MVFs may be that they are already delivered as microvessels. Although the use of freshly isolated MVFs bypasses the need for de novo microvessel formation and vessel infiltration from the host, there is still a period of time that needs to pass for network formation to occur with freshly isolated MVFs before this strategy can be fully effective.

In the pursuit of clinically relevant procedures for the treatment of VML, all constructs in the study were created on the day of implantation. ASCs were previously cultureexpanded to improve purity and to emulate an off-the-shelf stem cell product. In contrast, MVFs were isolated on the same day of surgery, and prior culturing to improve purity or allow for microvascular development in vitro was not necessary to realize a benefit. Allowing for the development of a more mature network in vitro may be a means to further improve the vascularization potential in vivo for both groups. With regards to the MVFs, allowing for the progression through angiogenic and neovascular remodeling phases in vitro (Shepherd et al., 2004; Nunes et al., 2010) may support improved inosculation and enable perfusion throughout the defect. Similarly, extending the in vitro culture of a prevascularized construct containing myoblasts, endothelial cells, and fibroblasts had a significant impact on blood vessel perfusion in vivo (Koffler et al., 2011). Conversely, it is worth considering that freshly isolated ASCs may improve their vascular potential. Both immunophenotype and VEGF secretion of mesenchymal stem cells (MSCs) are affected by passage leaving open the possibility that changes in the culture status of ASCs may have an effect on vessel development in vivo (Crisostomo et al., 2006; Mitchell et al., 2006). Nonetheless, the fact that MVFs were isolated and implanted on the day of surgery and provided superior vascularity over that of ASCs, at least under the conditions of the current study, provides enthusiasm for further exploration into their potential for VML and other muscle defects. 
With regards to skeletal muscle, prevascularization strategies have been extremely encouraging but have largely been limited to proof-of-concept studies using subcutaneous implants or relatively small muscle defects (Levenberg et al., 2005; Koffler et al., 2011). In the current study a relatively large volumetric muscle loss model was used to evaluate potential therapeutic strategies that can improve vascularity. Aside from the injury model used, another key distinction between the current strategy using MVFs and other prevascularization strategies is that the majority of them utilizes a combination of cells and requires extensive in vitro manipulation. For example, the transplantation of constructs containing endothelial cells, myoblasts, and fibroblasts that underwent vascular and myogenic development in vitro resulted in substantial tissue perfusion when implanted in an abdominal defect (Koffler et al., 2011). A key difference between a strategy like this and the use of MVFs is that instead of attempting to create microvessels, the microvessels procured from adipose tissue are already functional, at least just prior to their isolation. Although the isolation procedure may have some effects on cellular function, their ability to form networks after procurement is evidence that the MVFs maintain the requisite cells at the appropriate ratios (Hoying et al., 1996).

It is well-known that severe muscle injuries induce activation and participation of resident stem cells known as satellite cells which are critical for muscle healing (Schultz and McCormick, 1994). In regenerating muscle, myogenesis and angiogenesis occur in tandem. The observations that satellite cells positively impact microvascular fragment angiogenesis (Rhoads et al., 2009) provided evidence to suggest that MVFs would be well-suited for a large skeletal muscle defect. Others have also documented the interplay between vascular cells and satellite cells, a concept that is intuitive when considering the close anatomic association between vessels and satellite cells in vivo (Christov et al., 2007). The influence of resident stem cells, to include satellite cells, on the continued vascular growth between 7 and 14 days was not determined. Experiments utilizing Pax 7 transgenic mice where satellite cell activity is diminished (Sambasivan et al., 2011) would be valuable to conclusively determine the impact of myogenic cells on MVF angiogenesis in vivo. However, the presence of immature muscle fibers associated with microvessels in the MVF group (Fig. 5) is also consistent with the coordinated development of angiogenesis and myogenesis and is highly encouraging. Although the presence of immature muscle fibers was only measured relatively early in the current study, it alludes to the possibility that MVF transplantation may be a means to support continued muscle regeneration. Future mechanistic studies addressing the factors critical for MVF implant success and ways to further enhance their ability to support myogenesis represents a means to further improve the utility of MVFs for skeletal muscle.

In addition to the straightforward mechanism whereby MVFs inosculate with the host, we explored the possibility that MVFs may provide growth factors that support vasculogenesis. However, the levels of VEGF secreted by MVFs were significantly lower than that of ASCs under both normoxic and hypoxic conditions (Fig. 2), suggesting that the higher vascularity observed in vivo with MVFs, at least early after injury, was not due to an increased secretion of VEGF. As discussed above, since stem cells are associated with microvessels (Crisan et al., 2008), and MVFs contain cells with properties that are consistent with mesenchymal stem cells (MSCs) (Laschke et al., 2012), the possible influence of stem cells associated with the MVFs on angiogenesis should not be disregarded. The lack of significant vascular ingrowth from the host in COL and ASCs on day 7 (Figs. 3-5) support the contention that the bulk of the vascularity observed with MVFs was a direct result of implanted vasculature. To gain better insight into the mechanisms whereby large changes in vascularity were observed within VML defects, a followup study was completed using MVFs from GFP transgenic animals in order to identify the source of vessels (i.e., host or implant). GFP MVFs were clearly found within the defect, and the majority of the GFP positive vessels were also GS-Lectin positive (Figs. 8). Thus, the main source of vasculature when MVFs were used, were the MVFs that were transplanted. These findings are in agreement with previous reports where increases in vessel density with the use of MVFs were due to the transplantation of the isolate, as opposed to the ingrowth of host vessels (Shepherd et al., 2004). Some lectin ${ }^{+}$GFP- negative cells could be found within the defect at the interface. However, there was a high level of vascular activity that was observed at the interface when MVFs were derived from non-GFP animals, and was even observed in ASC and COL implants 7 days after injury (Fig. 2). More importantly, a high degree of vascularity was consistently observed within the defect with MVF implantation. This is a critical distinction since it is significantly more challenging to provide a vasculature to the center of large defects, away from the interface, a finding repeatedly observed with MVFs even at day 7, and by day 14 with ASCs.

Despite the high vascularity throughout the defect, the level of vessel perfusion was relatively low. This is in close agreement with previous studies where freshly isolated MVFs exhibited relatively low levels of perfusion in a subcutaneous implant (Nunes et al., 2010). The limited perfusion represents an area for which the use of freshly isolated MVFs can be improved upon. As discussed above, allowing for a more mature network development in vitro may be a solution; however, this eliminates the advantage of using a same-day surgical approach. Perhaps another approach may be the co-delivery of clinically relevant cells or growth factors that could augment the vascular potential of MVFs. Regardless, the data herein provide evidence that freshly isolated MVFs may serve as a platform for future studies to improve the healing of VML injuries.

A pertinent question that requires further investigation is whether improved vascularity early on from MVFs translates to improved functional outcomes. Despite large differences in vascularity among the groups and some evidence of improved myogenesis there were no differences in muscle mass (Table 1), presumably due to the early time point studied and the nature of the defect. Interestingly, the presence of immature muscle fibers in association with microvessels relatively early after the 
injury raises the possibility that early changes in muscle regeneration may have a beneficial impact on muscle repair later on. Overall, the current findings raise the intriguing possibility that improved vascularity with MVFs early after injury may translate to lesser fibrosis and improved outcomes at later time points, similar to that observed when using ASCs (Hwang et al., 2013; Park et al., 2012).

\section{Conclusions}

Given the large changes in vascularity observed with MVFs, it is plausible to suggest that their combination with other facets of tissue engineering where myogenic cells can be delivered, i.e., delivering them in a scaffold with or without growth factors may further enhance tissue perfusion and subsequent muscle regeneration. In the current study MVFs were delivered in a relatively standard collagen scaffold without any additional growth factors, creating a sound platform for future advances for the treatment of VML. Overall, the findings herein are encouraging and support the exploration of MVFs as a clinically relevant means to improve tissue perfusion for large musculoskeletal injuries.

\section{Acknowledgements}

This study was supported by the U.S. Army Medical Research and Materiel Command of the Department of Defense. The opinions or assertions contained herein are the private views of the authors and are not to be construed as official or reflecting the views of the Department of Defense or US government. This research was performed while the author (M.P) held a National Research Council Research Associateship Award at the United States Army Institute of Surgical Research. The authors would like to express their sincere gratitude to Monica Jalomo, Janet Roe, and Stephanie Roth for their invaluable technical assistance.

\section{References}

Cao Y, Sun Z, Liao L, Meng Y, Han Q, Zhao RC (2005) Human adipose tissue-derived stem cells differentiate into endothelial cells in vitro and improve postnatal neovascularization in vivo. Biochem Biophys Res Commun 332: 370-379.

Christov C, Chretien F, Abou-Khalil R, Bassez G, Vallet G, Authier FJ, Bassaglia Y, Shinin V, Tajbakhsh S, Chazaud B, Gherardi RK (2007) Muscle satellite cells and endothelial cells: close neighbors and privileged partners. Mol Biol Cell 18: 1397-1409.

Cierny G 3rd, Byrd HS, Jones RE (1983) Primary versus delayed soft tissue coverage for severe open tibial fractures. A comparison of results. Clin Orthop Relat Res 178: $54-63$.

Corona BT, Garg K, Ward CL, McDaniel JS, Walters TJ, Rathbone CR (2013a) Autologous minced muscle grafts: a tissue engineering therapy for the volumetric loss of skeletal muscle. Am J Physiol Cell Physiol 305: C761-C775.

Corona BT, Wu X, Ward CL, McDaniel JS, Rathbone CR, Walters TJ (2013b) The promotion of a functional fibrosis in skeletal muscle with volumetric muscle loss injury following the transplantation of muscle-ECM. Biomaterials 34: 3324-3335.

Crisan M, Yap S, Casteilla L, Chen CW, Corselli M, Park TS, Andriolo G, Sun B, Zheng B, Zhang L, Norotte C, Teng PN, Traas J, Schugar R, Deasy BM, Badylak S, Buhring HJ, Giacobino JP, Lazzari L, Huard J, Péault B (2008) A perivascular origin for mesenchymal stem cells in multiple human organs. Cell Stem Cell 3: 301-313.

Crisostomo PR, Wang M, Wairiuko GM, Morrell ED, Terrell AM, Seshadri P, Nam UH, Meldrum DR (2006) High passage number of stem cells adversely affects stem cell activation and myocardial protection. Shock 26: 575 580 .

Cross JD, Ficke JR, Hsu JR, Masini BD, Wenke JC (2011) Battlefield orthopaedic injuries cause the majority of long-term disabilities. J Am Acad Orthop Surg 19 Suppl 1: S1-7.

Deveci D, Marshall JM, Egginton S (2001) Relationship between capillary angiogenesis, fiber type, and fiber size in chronic systemic hypoxia. Am J Physiol Heart Circ Physiol 281: H241-252.

Ergun S, Tilki D, Klein D (2011) Vascular wall as a reservoir for different types of stem and progenitor cells. Antioxid Redox Signal 15: 981-995.

Grogan BF, Hsu JR (2011) Volumetric muscle loss. J Am Acad Orthop Surg 19 Suppl 1: S35-37.

Hoying JB, Boswell CA, Williams SK (1996) Angiogenic potential of microvessel fragments established in three-dimensional collagen gels. In Vitro Cell Dev Biol Anim 32: 409-419.

Hwang JH, Kim IG, Piao S, Jung AR, Lee JY, Park KD (2013) Combination therapy of human adipose-derived stem cells and basic fibroblast growth factor hydrogel in muscle regeneration. Biomaterials 34: 6037-6045.

Jabbarzadeh E, Starnes T, Khan YM, Jiang T, Wirtel AJ, DEng M, Lv Q, Nair LS, Doty SB, Laurencin CT (2008) Induction of angiogenesis in tissue-engineered scaffolds designed for bone repair: a combined gene therapy-cell transplantation approach. Proc Natl Acad Sci USA 105: 11099-11104.

Koffler J, Kaufman-Francis K, Shandalov Y, Egozi D, Pavlov DA, Landesberg A, Levenberg S (2011) Improved vascular organization enhances functional integration of engineered skeletal muscle grafts. Proc Natl Acad Sci USA 108: 14789-14794.

Kramers-de Quervain IA, Lauffer JM, Kach K, Trentz O, Stussi E (2001) Functional donor-site morbidity during level and uphill gait after a gastrocnemius or soleus muscleflap procedure. J Bone Joint Surg Am 83-a: 239-246.

Laschke MW, Kleer S, Scheuer C, Schuler S, Garcia P, Eglin D, Alini M, Menger MD (2012) Vascularisation of porous scaffolds is improved by incorporation of adipose tissue-derived microvascular fragments. Eur Cell Mater 24: 266-277. 
Laschke MW, Menger MD (2012) Vascularization in tissue engineering: angiogenesis versus inosculation. Eur Surg Res 48: 85-92.

Lesman A, Koffler J, Atlas R, Blinder YJ, Kam Z, Levenberg S (2011) Engineering vessel-like networks within multicellular fibrin-based constructs. Biomaterials 32: 7856-7869.

Levenberg S, Rouwkema J, Macdonald M, Garfein ES, Kohane DS, Darland DC, Marini R, van Blitterswijk CA, Mulligan RC, D'Amore PA, Langer R (2005) Engineering vascularized skeletal muscle tissue. Nat Biotechnol 23: 879-884.

Li MT, Willett NJ, Uhrig BA, Guldberg RE, Warren GL (2014) Functional analysis of limb recovery following autograft treatment of volumetric muscle loss in the quadriceps femoris. J Biomech 47: 2013-2021.

Li Y, Song Y, Zhao L, Gaidosh G, Laties AM, Wen R (2008) Direct labeling and visualization of blood vessels with lipophilic carbocyanine dye DiI. Nat Protoc 3: 1703 1708.

Linderman JR, Kloehn MR, Greene AS (2000) Development of an implantable muscle stimulator: measurement of stimulated angiogenesis and poststimulus vessel regression. Microcirculation 7: 119-128.

Markley JM, Faulkner JA, Carlson BM (1978) Regeneration of skeletal muscle after grafting in monkeys. Plast Reconstr Surg 62: 415-422.

Mitchell JB, McIntosh K, Zvonic S, Garrett S, Floyd ZE, Kloster A, Di Halvorsen Y, Storms RW, Goh B, Kilroy G, Wu X, Gimble JM (2006) Immunophenotype of human adipose-derived cells: temporal changes in stromalassociated and stem cell-associated markers. Stem Cells 24: 376-385.

Mizuno H (2010) The potential for treatment of skeletal muscle disorders with adipose-derived stem cells. Curr Stem Cell Res Ther 5: 133-136.

Nunes SS, Greer KA, Stiening CM, Chen HY, Kidd KR, Schwartz MA, Sullivan CJ, Rekapally H, Hoving JB (2010) Implanted microvessels progress through distinct neovascularization phenotypes. Microvasc Res 79: 10-20.

Park JK, Ki MR, Lee EM, Kim AY, You SY, Han SY, Lee EJ, Hong IH, Kwon SH, Kim SJ, Rando TA, Jeong KS (2012) Losartan improves adipose tissue-derived stem cell niche by inhibiting transforming growth factor-beta and fibrosis in skeletal muscle injury. Cell Transplant 21: 2407-2424.

Planat-Benard V, Silvestre JS, Cousin B, André M, Nibbelink M, Tamarat R, Clergue M, Manneville C, Saillan-Barreau C, Duriez M, Tedgui A, Levy B, Pénicaud L, Casteilla L (2004) Plasticity of human adipose lineage cells toward endothelial cells: physiological and therapeutic perspectives. Circulation 109: 656-663.

Rhoads RP, Johnson RM, Rathbone CR, Liu X, TemmGrove C, Sheehan SM, Hoying JB, Allen RE (2009) Satellite cell-mediated angiogenesis in vitro coincides with a functional hypoxia-inducible factor pathway. Am J Physiol Cell Physiol 296: C1321-1328.

Rieder MJ, O’Drobinak DM, Greene AS (1995) A computerized method for determination of microvascular density. Microvasc Res 49: 180-189.
Rossi CA, Flaibani M, Blaauw B, Pozzobon M, Figallo E, Reggiani C, Vitello L, Elvassore N, De Coppi $\mathrm{P}$ (2011) In vivo tissue engineering of functional skeletal muscle by freshly isolated satellite cells embedded in a photopolymerizable hydrogel. FASEB J 25: 2296-2304.

Sambasivan R, Yao R, Kissenpfennig A, Van Wittenberghe L, Paldi A, Gayraud-Morel B, Guenou H, Malissen B, Tajbakhsh S, Galy A (2011) Pax7-expressing satellite cells are indispensable for adult skeletal muscle regeneration. Development 138: 3647-3656.

Schultz E, McCormick KM (1994) Skeletal muscle satellite cells. Rev Physiol Biochem Pharmacol 123: 213257.

Shepherd BR, Chen HY, Smith CM, Gruionu G, Williams SK, Hoying JB (2004) Rapid perfusion and network remodeling in a microvascular construct after implantation. Arterioscler Thromb Vasc Biol 24: 898-904.

Shepherd BR, Hoying JB, Williams SK (2007) Microvascular transplantation after acute myocardial infarction. Tissue Eng 13: 2871-2879.

Zuk PA, Zhu M, Mizuno H, Huang J, Futrell JW, Katz AJ, Benhaim P, Lorenz HP, Hedrick MH (2001) Multilineage cells from human adipose tissue: implications for cell-based therapies. Tissue Eng 7: 211-228.

\section{Discussion with Reviewers}

R. Guldberg: Will accelerated re-establishment of perfusion in volumetric muscle injuries translate to improved functional muscle regeneration?

Authors: It is unknown at this time whether only the reestablishment of perfusion in large volumetric muscle loss injuries is sufficient to support the myogenesis required for functional muscle regeneration. Improving tissue perfusion leads to improved functional muscle regeneration in other skeletal muscle injury models (e.g., ischemia), however, in such models the repair of myofibers is supported by a supply of myogenic cells that are in close proximity to the injured tissue. Although our results suggest that improving vascularization and perfusion has the potential to improve myogenesis within volumetric muscle loss defects at early time points, whether this results in the generation of a sufficient number of functional myofibers over time requires further study.

C.T. Laurencin: This experimental study convincingly demonstrates that the transplantation of microvascular fragments is a promising strategy to improve the vascularization of large volumetric muscle loss defects. Can the authors estimate which amount of fat tissue for the isolation of these fragments would be necessary to apply this strategy in patients under clinical conditions?

Authors: In the current study approximately 1.6-2.0 x $10^{4}$ MVFs were used to replace a $\sim 0.95 \mathrm{~mL}$ TA VML defect $(0.95 \mathrm{~mL}=1.05 \mathrm{~g} / \mathrm{mL}$ skeletal muscle density x $0.1 \mathrm{~g}$ defect). If the objective is to repair a $28.6 \mathrm{~mL}$ defect $(20 \%$ of a $143 \mathrm{~mL}$ human TA (Fukunaga et al., 1992, additional reference)) $\sim 6.0 \times 10^{6}$ MVFs would be required. A conservative yield of MVFs from adipose tissue is roughly 
$6 \times 10^{3} \mathrm{MVFs} / \mathrm{g}$ adipose tissue. To replace a $20 \%$ human TA VML defect approximately $1 \mathrm{~kg}$ of adipose tissue would be necessary.

M. Laschke: Did the augmentation of perfusion produce any histological or functional improvements in muscle regeneration in the VML model?

Authors: This is an important point to be considered since the ultimate goal of a VML therapy is to improve muscle regeneration and function. Our primary objective in the current study was to promote an early vascularization; given the severity of the VML injury, the beneficial effects (muscle regeneration and function) of an early therapy may not be evident until several months later. We did see some signs of early muscle regeneration providing enthusiasm that functional outcomes may be improved at later time points. This is an important concept that will be addressed in future studies.

M. Laschke: How rapidly is the perfusion status reestablished in the graft center with this approach given that the implanted MVFs must form an early network and inosculate with the host circulation? How will such delays and heterogeneity of reperfusion, if any, impact the use of this approach in cell seeded constructs?
Authors: The rapidity at which inosculation occurs when using freshly isolated MVFs has not been tested in this VML model. Others have demonstrated inosculation after one day when grafts were implanted after murine myocardial infarction (Shepherd, 2007, text reference). However, caution should be made when extrapolating these findings to the current study due to the differences between the models (myocardial infarction vs. VML) and the time allowed for microvessel network development (7 days $v s$. freshly isolated). Presumably, an earlier inosculation would support the cell survival of cells co-transplanted with MVFs. If a delay in reperfusion significantly diminishes the survival of co-transplanted cells it may be more prudent to consider allowing for a more mature network to develop in vitro prior to construct implantation and/or providing an additional stimulus to accelerate network formation.

\section{Additional Reference}

Fukunaga T, Roy RR, Shellock FG, Hodgson JA, Day MK, Lee PL, Kwong-Fu H, Edgerton VR (1992) Physiological cross-sectional area of human leg muscles based on magnetic resonance imaging. J Orthop Res 10: 928-934. 